\title{
CIBERESPAÇO: CAMINHOS ABERTOS A NOVAS INTERAÇÕES DOCENTES *
}

\author{
Vera Cristina Queiroz
}

$* *$

\section{Resumo}

O presente trabalho é fruto de uma pesquisa de doutorado, cuja tese foi concluída em abril de 2005. O trabalho tem por objetivo apresentar os resultados de pesquisa sobre uma vivência de docência colaborativa on-line que abrange diversos aspectos dessa docência: tipos e número de provocações didáticas propostos pelas professoras; interações colaborativas realizadas entre as professoras; respostas dos alunos às provocações para realização de tarefas colaborativas, e aprendizagens docente e discente resultantes. Por questões de complexidade desses aspectos e de tempo para discorrer sobre cada um deles, neste trabalho elegemos apresentar os resultados da vivência de uma prática de atuação conjunta entre professores on-line.

Palavras-chave: docência colaborativa on-line; interações entre professores on-line; tecnologia on-line.

\section{Abstract}

The present work is based on a PhD research concluded in April 2005. The work has as objective to present the research results of an on-line teachers' collaborative experience that encompasses several aspects of teaching: types and numbers of didactic challenges proposed by the teachers; collaborative interactions among the teachers; students' answers to challenges to perform the collaborative tasks, and the resulting teachers' and students' learnings. Due to the complexity of these aspects and to the time to discuss each one of them, in this article we have chosen to present the results of the experience of a practice of collaborative work between on-line teachers.

Key words: on-line collaborative teaching; interaction between on-line teachers; on-line technology.

\section{Introdução}

As transformações geradas pelo fenômeno da globalização, incrementado pelos recursos tecnológicos, provocam mudanças significativas no comportamento da sociedade contemporânea, uma vez que possibilitam e incentivam as interações entre os seres humanos, até mesmo entre aqueles que se encontram geograficamente distantes e são oriundos de diferentes culturas.

$\mathrm{O}$ acesso a profissionais de áreas correlatas e afins ou de outras áreas de estudo/conhecimento para trocas de informações, compartilhamento e/ou aquisição de novos saberes se torna mais rápido, fácil e viável através dos recursos tecnológicos e da difusão da rede mundial de computadores.

\footnotetext{
* Trabalho extraído de tese

* * Doutora em Educação pela FEUSP, profissional autônomo, consultora em EAD e palestrante, vcqm@uol.com.br;

URL: http://www.geocities.com/vcqm/
} 
Torna-se possível, então, a interação e a comunicação a qualquer hora/dia com pessoas de qualquer parte do mundo, o que amplia as possibilidades de exploração de novas experiências interpessoais, profissionais, culturais e educacionais, e o estabelecimento de novas formas de sociabilidade.

Em estudos sobre espaço cibernético, é possível verificar que novas formas de sociabilidade estão sendo engendradas. Fundamenta Guimarães Jr. (1997), por exemplo, que "o ciberespaço, ao constituir-se em um novo espaço de sociabilidade, acaba gerando novas formas de relações sociais, com códigos e estruturas próprias”.

Esta afirmação de Guimarães Jr. talvez se dê pelo fato de que os espaços virtuais podem reunir pessoas que não têm contato pessoal fora da rede. Inclusive, podem agregar indivíduos de culturas totalmente diversas e que jamais poderiam interagir se não fosse através das possibilidades de superação dos limites de espaço e tempo, proporcionados pelas tecnologias digitais.

A respeito das novas formas de interação e de sociabilidade possibilitadas pelas redes telemáticas, Rodrigues (1994, apud MARCELO 2001, p. 65) afirma que " $a$ comunicação não é um produto, mas um processo de troca simbólica generalizada, processo de que se alimenta a sociabilidade que gera os laços sociais que estabelecemos com os outros".

Percebe-se, desta forma, o importante papel da tecnologia na vida dos cidadãos da contemporaneidade: quer no estabelecimento de relações pessoais, culturais ou profissionais, quer na concretização de condutas de partilha e de uma nova sociabilidade.

Apesar de a tecnologia ampliar possibilidades de interação/comunicação entre os indivíduos, é preciso vê-la apenas como um meio e não como um fim. Torna-se imperativo, pois, que o ser humano aprenda a viver, se comunicar e compartilhar e se projete além de suas origens/raizes culturais, buscando se tornar assim um indivíduo planetário (MORIN, 2000).

\section{A Pesquisa}

Levando em conta a relevância do intercâmbio de idéias entre profissionais das mais diversas áreas do conhecimento e dentro de áreas afins, realizamos uma pesquisa de doutorado, cuja tese foi concluída em abril de 2005, em que apresentamos os resultados de uma vivência de docência colaborativa on-line. Esses resultados se distribuem entre diversos aspectos abrangidos pela docência colaborativa, tais como: tipos e número de provocações didáticas propostos pelas professoras; interações colaborativas realizadas entre as professoras; respostas dos alunos às provocações para realização de tarefas colaborativas, e aprendizagens docente e discente resultantes.

Embora não ignoremos a importância e complexidade e a relação de complementaridade entre cada um desses aspectos, elegemos apresentar no presente trabalho apenas uma das vertentes dessa temática, ou seja, os resultados de pesquisa sobre a atuação colaborativa de professores aberta e possibilitada pelo ciberespaço.

$\mathrm{O}$ objeto da pesquisa foi um curso experimental de inglês básico on-line para alunos de diferentes países, cuja língua materna não é o inglês e que adotava uma metodologia de ensino-aprendizagem colaborativa/comunicacional. Tal metodologia de ensino baseou-se principalmente em estratégias didáticas que buscavam enfatizar a construção compartilhada do conhecimento, a interação, colaboração e a troca de experiências entre os sujeitos envolvidos na aprendizagem (professores e alunos). 
O curso, em seu primeiro momento de realização (de janeiro a meados de julho de 2001), foi ministrado concomitantemente por duas professoras da área de Língua Inglesa (eu e uma professora natural da e residente na Argentina), tendo em vista oferecer maior suporte lingüístico e comunicacional aos alunos.

\section{Interações Colaborativas entre Professores On-line}

A solicitação que recebi da professora argentina ${ }^{1}$ sobre organização de cursos on-line, encaminhou-me a acolhê-la no curso experimental de meu projeto de pesquisa, dividindo com ela encargos didáticos. A partir do motivo que encaminhara sua solicitação de participação, esclareceram-se dois papéis docentes: o de professora colaboradora - sugerido por ela e por mim a ela atribuído -, e o de professora pesquisadora, por mim desenvolvido nessa experiência.

A ação docente conjunta se deu pelo fato de eu querer testar a possibilidade de exercer a docência colaborativa à distância, o que para mim, de modo particular, se configurava como uma prática educacional desafiadora. Quanto à professora argentina o interesse estava na organização de cursos on-line. É importante ressaltar que apesar de o curso ter sido ministrado a quatro mãos, o planejamento metodológico não foi discutido entre nós professoras, uma vez que já havia sido feito por mim quando do contato da professora argentina comigo.

No desenrolar da pesquisa, a professora argentina se concentrou na elaboração de atividades didáticas que eram, então, discutidas por nós em sessões de chat ou submetidas a mim via $e$-mail para apreciação, comentários e re-estruturação consensual (quando julgávamos necessário).

A mim coube, atuar como coordenadora responsável pelo design do curso; pela administração do ambiente virtual no qual o curso se inseriu; pela orientação dos alunos quanto a problemas técnicos básicos de acesso ao ambiente, e na elaboração da proposta didática e acompanhamento do processo de ensino-aprendizagem, em todas as suas etapas, do planejamento à avaliação.

Para que essa docência fosse possível, uma vez que se realizava à distância, mantivemos constantes contatos através do meio eletrônico (computador/Internet).

Foram trocados ao todo 123 e-mail entre as professoras, sendo 52 por mim a ela enviados, e 71 a mim enviados pela professora argentina.

Concomitantemente à troca de e-mail, agendamos sessões de chat para (em tempo real) discutir, principalmente, as atividades do curso. Foram realizadas 17 sessões de chat no $\mathbf{I C Q}^{2}$ e 1 no Blackboard ${ }^{3}$. Esta última (em 17 de fevereiro de 2001) visava especificamente apresentar a ferramenta de chat do Blackboard para a professora argentina, e com isso viabilizar o agendamento de uma sessão de chat entre nós professoras e os alunos, utilizando essa ferramenta.

A frequiência dos contatos entre as professoras, além de facilitar o trabalho colaborativo, também estabeleceu vínculos entre nós que nos permitiram nos expressarmos livremente a respeito das propostas didáticas apresentadas por uma ou pela outra, como se verificam nos seguintes excertos:

\footnotetext{
${ }^{1} \mathrm{O}$ contato da professora argentina comigo se deu através de um coordenador de um grupo de professores voluntários que ministram cursos de inglês on-line.

${ }^{2}$ ICQ (do inglês I seek you, eu procuro você) é um programa bastante popular, pois permite a conversação 1 a 1 ou em pequenos grupos, mandar mensagens instantâneas, e o envio e recebimento de arquivos (documentos, imagens e sons). Além disso, permite a seus usuários ver se outros usuários do mesmo programa estão conectados à Internet.

${ }^{3}$ Plataforma de gerenciamento de cursos na Web escolhida para acomodar o curso.

V.3 $\mathrm{N}^{\circ} 2$, Novembro, 2005
} 
To: Professora argentina

From: Eu

Subject: Re:Classes At 18:17 10/02/01 -0300, you wrote:

Hi!,

Here im sending the files for the sentences and conjunctions. Tomorrow I will send the other for verbs. I was thinking to post them at blackboard and then when they have the answers the can send them to my mail. I will correct them and then I will send them to you just to have a look. Do you agree? So in this way we could go directly to their problems.

I just loved the quizzes. If you have any problems in uploading the docs in the assignments area, please let me know. I can easily post them there.

As for correction, we could place an answer key linked to the quiz at Blackboard and ask students to email us only the problems they had. This way we would reinforce the idea of having independent learners who are responsible for their own learning process. What do you think?

Great!!!

After they do all these quizzes, they will have to gather in threes and with the ideas from the sentences they have to write a WELL PLANNED PARAGRAPH. But this is next step. Do you agree?

Definitely!!!!! :-)

From: "Professora argentina"

To: "Eu"

Subject: RE: news

Professora argentina: What you are doing on blackboard is great. Now im sending you the objective for the first unit.. we can call it THE SIMPLE SENTENCE

OBJECTIVE: Write grammatically correct simple sentences

Task nro. 1: a) what do you understand by "Parts of Speech"? b) How many parts of speech are there in the English language?

Eu: They will be able to move forward when then answer to this in the correct way ok? Do you agree?

OK THIS WILL BE THE WAY IN WHICH I THOUGHT TO DO THE COURSE FOCUSING ON WRITING OK? Then I will send them exercises. Then we will correct together or we will see. 
Hugs,

As expressões de incentivo (great, definitely, so do $I$ ) bem como de questionamento de opinião (OK?, What do you think?, Do you agree?) encontradas nas mensagens são sinalizadoras de trocas horizontalizadas entre as professoras. Essas trocas foram fundamentais para que o intercâmbio profissional se estabelecesse apesar de a professora argentina e eu não nos conhecermos pessoalmente.

A experiência em ministrar um curso com a professora argentina foi bastante rica, motivadora e gratificante, porque permitiu experienciar/vivenciar a viabilização de ensino colaborativo à distância, com professores separados geograficamente e também de culturas diferentes. Essa experiência contribui para nosso desenvolvimento profissional e humano, pois como adverte Lévy (1998a, p. 27) "toda atividade, todo ato de comunicação, toda relação humana implica um aprendizado".

O desafio da docência conjunta (principalmente a distância) está na articulação clara, objetiva e sincera entre as professoras. Qualquer mal-entendido que possa vir a ocorrer entre as educadoras deve ser imediatamente analisado, elucidado e trabalhado conjuntamente para reforçar os vínculos da colaboração docente e, desta forma, não comprometer a qualidade do processo de ensino.

A ocorrência de situação conflitante na relação interprofissional entre as professoras foi identificada, o que é mostrado no exemplo a seguir, onde a professora argentina questiona a validade de eu querer expor aos alunos os objetivos das atividades didáticas propostas (Regarding the objectives I think it is great to have them in mind. [...] But I don't think it is necessary to notify our students...):

\author{
From: "professora argentina" \\ To: "Eu" \\ Subject: RE: Assignments \\ Date: Mon, 2 Apr 2001 11:40:43 -0300 \\ Dear,
}

Regarding the objectives I think it is great to have them in mind. This is the first thing I do when I plan a class. But I don`t think it is necessary to notify our students. They only have to feel they are LEARNING.

Objectives and thesis or studies we are doing are of our exclusive business Vera. So it is ok that we exchange ideas on how to deliver a class or develop a course but not being to bossy to make them realize they are our guinea pigs. They have just to feel their progress, we can tell them..."This exercise is for you to improve grammar points, or language" but not pointing out OUR objectives, they are not interested in our pedagogy to let them learn... < snip $>$ 
O questionamento a respeito do meu procedimento didático foi causa de constrangimento e me fez considerar uma possível resistência, ou desconhecimento de certos princípios da metodologia colaborativa/comunicacional, ou indisposição decorrente de mudanças do comportamento docente da professora argentina, e não compartilhada comigo. Isto porque na perspectiva dessa metodologia, adotada no curso, o ensino é uma relação sócio-pedagógica entre os sujeitos (professores e alunos) nela envolvidos. Portanto, os objetivos didáticos pretendidos com o trabalho docente devem ser compartilhados com os alunos, possibilitando com isso integrá-los na situação de ensino da qual fazem parte.

Se procedentes as considerações anteriores é possível supor que os princípios metodológicos adotados estavam talvez mais claros para mim do que para a professora argentina. De qualquer modo é preciso entender que ambas incursionávamos por novos caminhos educacionais. Desta forma, tais deslizes são indicadores de um percurso de transição do ensino tradicional para o ensino colaborativo/comunicacional.

Julgo também relevante mencionar que a forma pela qual a mensagem foi redigida pela professora argentina acarretou em mim mal estar. A valorização das palavras LEARNING, OUR escritas em letras maiúsculas e o uso de expressões como our exclusive business (o que diz respeito somente a nós), bossy (autoritária) e guinea pigs (cobaias) para se referir aos alunos-, foram por mim interpretadas como rudes, ofensivas e agressivas.

A constatação de tal ocorrência exigia esclarecimento urgente, o que resultou no envio da seguinte mensagem para a professora argentina:

To: Professora argentina

From: Eu

Subject Re: Assignments. Date: Mon, 2 Apr 2001

Dear,

Sorry, I did not want to be bossy, nor did I want to make them our guinea pigs. The idea of setting objectives was only to make them feel that the course was more academic, that it was carefully prepared for them. IMHO, having the objectives set won't interfere negatively in their learning. Also, in an online course I believe everything has to be well structured. If you think I did something wrong, please forgive me. Perhaps I was extra enthusiastic :-(

If you totally disagree upon my initiative, I'll remove all that.

....

I spent two days trying to make the site look better, more attractive, more didactic. I tried to show that they are the ones who can learn by doing... that is what I believe... collaboration, interaction, participation in everything...

I agree that we should lead the course, and I guess we are doing it. Mistakes may happen sometimes... I do feel awful now. I want to do my best for the students, and I'm sure I've always done so. I really do not want to screw things up. Sorry, again.

[ ]s 
Através dessa mensagem, eu me desculpava por qualquer atitude autoritária assumida, tentava esclarecer o motivo pelo qual havia proposto colocar para os alunos o objetivo didático da elaboração da atividade, e expus o sentimento de embaraço no qual me encontrava, buscando transparência em nossas relações profissionais. Ao me posicionar, esperava a resolução da questão conflitante, o que de fato ocorreu com a mensagem (resposta) enviada pela professora argentina.

From: "Professora argentina"

To: "Eu"

Subject: no mistakes... our page is great!! Date: Mon, 2 Apr 2001 20:18:23 -0300

Dear Dear Dear !!!!!!,

Please I don't apologize in this way!!! Im learning from you, you are the expert!!!! So please don't make me feel bad. Im just exchanging ideas, WE are friends and partners. AND WE ARE BOTH DOING OUR BEST! We are both mistakes!!!! So please stop to blame on you!!!!

you will make me get mad!!:-)))))))))

I love the job we are doing!!! it is a great job. Only that we have to check all we do!!! and tell all!! in this way we will make a great page!!

WE ARE DOING IT!!! and think that you are the expert!!!! you are the one who is giving the page the professional look!! I don`t know how to do it!!! You have to teach me .

Ok. So don't change anything in the page!!! just keep on working. The only thing we have to check now is why some students are so quiet!! What can we do for them to react? Do you have any idea Vera? How about inviting them to a chat session on Sat? or probably you have any other idea. LET`S PUT OUR MINDS TOGETHER AND THINK :-) A BIG BIG KISS.

Com isso, fica evidenciada a preocupação da professora argentina com a resolução do incidente (Please, don't apologize this way!!!, I'm just exchanging ideas) e o seu interesse em continuar atuando no curso (And we are both doing our best; I love the job we are doing; Let's put our minds together and think).

Através da forma como a ocorrência conflitante foi encaminhada e de seu pronto esclarecimento foi possível reverter o quadro de desconforto criado entre as professoras e a retomada do trabalho docente conjunto.

Interpretando a ocorrência da situação conflitante, ocorrida entre nós professoras, à luz das assertivas de Lévy (1998b) e de Palloff e Pratt (1999), podemos afirmar que o conflito contribuiu para o estreitamento das nossas relações pessoais e profissionais e da qualidade do processo de ensino partilhado por nós. De onde se infere que o conflito explicitado e assumido é um sintoma de relações educacionais vivas, intencionadas, e não burocráticas. É indicador mesmo da incorporação de uma metodologia comunicacional de ensino, o que implica em transparência nas relações profissionais comprometidas com os objetivos educacionais propostos.

O conflito permitiu-me perceber ainda a importância da partilha dos fundamentos teórico-metodológicos do curso com o parceiro docente, desde o início dos trabalhos, ocasião em que os princípios e valores da docência seriam assumidos conjuntamente.

- $\quad$ A vivência da atuação docente conjunta aberta pelo ciberespaço nos mostrou que essa atuação é possível se nos orientarmos pelo princípio de alteridade, 
buscando atuar, somando as experiências profissionais; dispostos a lidar com as emoções que possam nos sobressaltar durante o percurso, e a encarar o papel do outro docente como o de um colaborador (conforme advoga LÉVY, 1998a).

Referências

GUIMARÃES Jr., M. J. L. A cibercultura e o Surgimento de Novas Formas de Sociabilidade. In: II REUNIÓN DE ANTROPOLOGIA DEL MERCOSUR. GT Nuevos mapas culturales: Cyber espacio y tecnologia de la virtualidad, Piriápolis, Uruguai, 1997. Disponível em <http://www.cfh.ufsc.br/ guima/>. Acesso em 10 jul. 2002.

LÉVY, P. A inteligência coletiva: por uma antropologia do ciberespaço. Trad. Luiz P. Rouanet. São Paulo: Loyola, 1998a.

1998b.

O que é o Virtual. 2. reimp., Trad. Paulo Neves. São Paulo: Editora 34 Ltda,

MARCELO, A. S. Internet e as Novas Formas de Sociabilidade. 2001. 159 p.

Dissertação de Mestrado em Ciências da Comunicação. Universidade da Beira Interior, Covilhã, Portugal. Disponível em: <http://www.bocc.ubi.pt/pag/marcelo-ana-sofiainternet-sociabilidade.pdf>. Acesso em: 25 abr. 2004.

MORIN, E. Os Sete Saberes Necessários à Educação do Futuro. Trad. de Catarina Elionora F. da Silva e Jeanne Sawaya, ver. téc. de Edgard de Assis Carvalho. SP: Cortez, Brasília, DF: UNESCO, 2000.

PALLOFF, R. M. \& PRATT, K. Building Learning Communities in Cyber-space: Effective Strategies for the Online Classroom. S.Francisco: Jossey-Brass Publishers, 1999. 